Research Article

\title{
On the Convergence of the Uniform Attractor for the 2D Leray- $\alpha$ Model
}

\author{
Gabriel Deugoué \\ Department of Mathematics and Computer Science, University of Dschang, P.O. Box 67, Dschang, Cameroon \\ Correspondence should be addressed to Gabriel Deugoué; agdeugoue@yahoo.fr
}

Received 15 January 2017; Revised 30 March 2017; Accepted 16 April 2017; Published 17 May 2017

Academic Editor: Julio D. Rossi

Copyright (C) 2017 Gabriel Deugoué. This is an open access article distributed under the Creative Commons Attribution License, which permits unrestricted use, distribution, and reproduction in any medium, provided the original work is properly cited.

We consider a nonautonomous 2D Leray- $\alpha$ model of fluid turbulence. We prove the existence of the uniform attractor $\mathscr{A}^{\alpha}$. We also study the convergence of $\mathscr{A}^{\alpha}$ as $\alpha$ goes to zero. More precisely, we prove that the uniform attractor $\mathscr{A}^{\alpha}$ converges to the uniform attractor of the 2D Navier-Stokes system as $\alpha$ tends to zero.

\section{Introduction}

In the past decades, the study of nonautonomous dynamical systems has been paid much attention as evidenced by the references cited in [1-8]. In [9], the author considers some special classes of nonautonomous dynamical systems and studies the existence and uniqueness of uniform attractors. In [10], the authors present a general approach that is well suited to construct the uniform attractor of some equations arising in mathematical physics (see also [11, 12]). In this approach, instead of considering a single process associated with the dynamical system, the authors consider a family of processes depending on a parameter (symbol) $\sigma$ in some Banach space. The approach preserves the leading concept of invariance, which implies the structure of the uniform attractors.

In this article, we study the following nonautonomous $2 \mathrm{D}$ Leray- $\alpha$ model:

$$
\begin{aligned}
\frac{\partial v}{\partial t}-v \Delta v+(u \cdot \nabla) v+\nabla p & =g_{0}(x, t), \\
v & =u-\alpha^{2} \Delta u, \\
\nabla \cdot u & =0, \\
\nabla \cdot v & =0, \\
v(\tau) & =v_{\tau},
\end{aligned}
$$

where $u$ is the velocity vector field, $p$ is the pressure, and $v$ is the viscosity coefficient. The spatial variable $x$ belongs to the two-dimensional torus $\mathbb{T}^{2}=[0,2 \pi L]^{2}$ and $\alpha$ is a parameter. Precise assumptions on the external force $g_{0}$ are given below. Formally, the above system is the 2D Navier-Stokes system when $\alpha=0$.

The $2 \mathrm{D}$ Leray- $\alpha$ model has received much attention over the past years (see [13] and the references therein) because of its importance in the description of fluid motion and turbulence. The 3D version of (1), namely, the 3D Leray- $\alpha$ model, was considered in [14] as a large eddy simulation subgrid scale model of 3D turbulence. In [15], the authors studied the relations between the long-time dynamics of the 3D Leray-alpha model and the 3D Navier-Stokes system. They found that bounded sets of solutions of the 3D Leray- $\alpha$ model converge to the trajectory attractor of the $3 \mathrm{D}$ Navier-Stokes system as time tends to infinity and $\alpha$ approaches zero. In particular, they showed that the trajectory attractor of the $3 \mathrm{D}$ Leray- $\alpha$ model converges to the trajectory attractor of the $3 \mathrm{D}$ Navier-Stokes system. In [16], analogous results were proven for the 3D Navier-Stokes- $\alpha$ model. In [17], the authors studied the convergence of the solution of the 2D stochastic Leray$\alpha$ model to the solution of the stochastic 2D Navier-Stokes equations as $\alpha$ approaches 0 . In particular, they proved the convergence in probability with the rate of convergence at most $O(\alpha)$.

The 2D Leray- $\alpha$ model has been studied analytically in [18] and computationally in [13]. In [18], the authors 
investigated the rate of convergence of four alpha models (2D Navier-Stokes- $\alpha$ model, 2D Leray- $\alpha$ model, 2D modified Leray- $\alpha$ model, and 2D simplified Bardina model) in the 2D case subject to periodic boundary conditions. In particular, they showed upper bounds in terms of $\alpha$ for the difference between solutions of the 2D $\alpha$-models and solutions of the 2D Navier-Stokes system. They found that all the four $\alpha$-models have the same order of convergence and error estimates. We also note that the autonomous and nonautonomous 2D Navier-Stokes- $\alpha$ models were considered in $[6,19]$. In [19], they proved that the global attractors of the 2D Navier-Stokes- $\alpha$ model converge to a subset of the global attractor of the 2D Navier-Stokes system when $\alpha$ approaches 0 . In [6], the authors studied the convergence of the uniform attractors of the 2D Navier-Stokes- $\alpha$ model when $\alpha$ tends to zero. They found that the uniform attractors of the 2D Navier-Stokes- $\alpha$ model converge to the uniform attractor of the 2D Navier-Stokes system when $\alpha$ approaches zero.

The purpose of this paper is to prove analogous results for the nonautonomous 2D Leray- $\alpha$ model. More precisely, we prove that the uniform attractors for the $2 \mathrm{D}$ Leray- $\alpha$ model converge to the uniform attractor of the 2D NavierStokes system when $\alpha$ approaches zero (see Theorem 13). Uniform attractors are not invariant under the family of processes; this brings about some difficulties in proving upper semicontinuous property. The proof of the convergence of the uniform attractors of the 2D Leray- $\alpha$ model uses the structure of uniform attractors which says that each uniform attractor is a union of kernels.

The article is structured as follows. In Section 2, we recall some properties of the uniform attractor for the $2 \mathrm{D}$ Navier-Stokes equations. In Section 3, we prove the existence and the structure of the uniform attractor of the 2D Leray$\alpha$ model. In Section 4, we prove the convergence of the uniform attractors of the 2D Leray- $\alpha$ model to the uniform attractor of the 2D Navier-Stokes system as $\alpha$ approaches zero.

\section{The 2D Navier-Stokes System and Its Uniform Attractor}

We consider the nonautonomous 2D Navier-Stokes system with periodic boundary conditions:

$$
\begin{aligned}
\frac{\partial u}{\partial t}-v \Delta u+(u \cdot \nabla) u+\nabla p & =g_{0}(t, x), \\
\nabla \cdot u & =0 .
\end{aligned}
$$

In (2), $u=u(x, t)=\left(u_{1}(x, t), u_{2}(x, t)\right)$ is the unknown vector field in $\mathbb{T}^{2}$ describing the motion of the fluid. The scalar function $p(x, t)$ is the unknown pressure and $g_{0}(x, t)$ is a given field of external force. Let $\mathscr{F}$ be the set of trigonometric polynomials of two variables with periodic domain $\mathbb{T}^{2}$ and spatial average zero; that is, for every $\Phi \in \mathscr{F}, \int_{\mathbb{T}^{2}} \Phi(x) d x=0$. We then set

$$
\mathscr{V}=\left\{\Phi \in \mathscr{F}^{2}: \nabla \cdot \Phi=0\right\} .
$$

We denote by $H$ and $V$ the closure of $\mathscr{V}$ in $L^{2}\left(\mathbb{T}^{2}\right)^{2}$ and $H^{1}\left(\mathbb{T}^{2}\right)^{2}$, respectively. The norms in $H$ and $V$ are denoted, respectively, by $|\cdot|$ and $\|\cdot\|$.

We denote by $\mathscr{P}: L^{2}\left(\mathbb{T}^{2}\right)^{2} \rightarrow H$ the HelmholtzLeray orthogonal projection operator and by $A=-\mathscr{P} \Delta$ the Stokes operator, subject to periodic boundary conditions, with domain $D(A)=H^{2}\left(\mathbb{T}^{2}\right)^{2} \cap V$. We note that in the space periodic case

$$
A=-\mathscr{P} \Delta=-\Delta .
$$

The operator $A^{-1}$ is a self-adjoint positive definite compact operator from $H$ into $H$. By $0<(2 \pi / L)^{2}=\lambda_{1} \leq \lambda_{2} \leq \cdots$, we denote the eigenvalues of $A$ in the $2 D$ case. It is well known that, in two dimensions, the eigenvalues of operator $A$ satisfy Weyl's type formula (see, e.g., $[13,15]$ ); namely, there exists a constant $c_{0}>0$ such that

$$
\frac{j}{c_{0}} \leq \frac{\lambda_{j}}{\lambda_{1}} \leq c_{0} j \quad \text { for } j=1,2, \ldots
$$

By

$$
\begin{aligned}
((u, v)) & =\left(A^{1 / 2} u, A^{1 / 2} v\right)=(\nabla u, \nabla v), \\
\|u\| & =\left|A^{1 / 2} u\right|
\end{aligned}
$$

for $u, v \in V$,

we denote the scalar product and the norm in $V$, respectively. Let $V^{\prime}$ be the dual space of $V$. For every $v \in V^{\prime}$, we denote by $\langle v, u\rangle$ the value of the functional $v$ from $V^{\prime}$ on a vector $u \in V$. The operator $A$ is an isomorphism from $V$ to $V^{\prime}$. In particular $((w, u))=\langle A w, u\rangle$ for all $w, u \in V$.

The Poincaré inequalities read

$$
\begin{aligned}
|u|^{2} \leq \lambda_{1}^{-1}\|u\|^{2}, & \forall u \in V, \\
\|u\|_{V^{\prime}}^{2} \leq \lambda_{1}^{-1}|u|^{2}, & \forall u \in H .
\end{aligned}
$$

For every $w_{1}, w_{2} \in \mathscr{V}$, we define the bilinear operator

$$
B\left(w_{1}, w_{2}\right)=\mathscr{P}\left(\left(w_{1} \cdot \nabla\right) w_{2}\right) .
$$

In the following lemma, we list certain relevant inequalities and properties of $B$ (see, e.g., [11]).

Lemma 1. The bilinear operator B defined in (9) satisfies the following.

$B$ can be extended as a continuous bilinear map $B: V \times$ $V \rightarrow V^{\prime}$. In particular, $B$ satisfies the following inequalities:

$$
\begin{array}{r}
\left|\langle B(u, v), w\rangle_{V^{\prime}}\right| \leq c|u|^{1 / 2}\|u\|^{1 / 2}\|v\||w|^{1 / 2}\|w\|^{1 / 2} \\
\forall u, v, w \in V, \\
\left|\langle B(u, v), w\rangle_{V^{\prime}}\right| \leq c|u|^{1 / 2}\|u\|^{1 / 2}|v|^{1 / 2}\|v\|^{1 / 2}\|w\| \\
\forall u, v, w \in V, \\
|(B(u, v), w)| \leq c\|u\|_{\infty}\|v\||w|, \\
\forall u \in D(A), v \in V, w \in H,
\end{array}
$$




$$
\begin{gathered}
|(B(u, v), w)| \leq c|u|\|\nabla v\||w|, \\
\forall u \in H, v \in D\left(A^{3 / 2}\right), w \in H, \\
\left|\langle B(u, v), w\rangle_{D(A)^{\prime}}\right| \leq c|u|\|v\|\|w\|_{\infty}, \\
\forall u \in H, v \in V, w \in D(A) .
\end{gathered}
$$

Moreover, for every $w_{1}, w_{2}, w_{3} \in V$, we have

$$
\left\langle B\left(w_{1}, w_{2}\right), w_{3}\right\rangle_{V^{\prime}}=-\left\langle B\left(w_{1}, w_{3}\right), w_{2}\right\rangle_{V^{\prime}},
$$

and in particular

$$
\left\langle B\left(w_{1}, w_{2}\right), w_{2}\right\rangle_{V^{\prime}}=0 .
$$

We apply the operator $\mathscr{P}$ to both sides of (2) and obtain an equivalent system:

$$
\frac{\partial u}{\partial t}+v A u+B(u, u)=g_{0}(x, t)
$$

The initial condition is posed at $t=\tau, \tau \in \mathbb{R}$ :

$$
u(\tau)=u_{\tau} \in H
$$

In order to clarify the assumptions on the external force $g_{0}$, we introduce the following notation. Given a Banach space $X$, we denote by $L_{b}^{2}(\mathbb{R} ; X)$ the subspace of $L_{\text {loc }}^{2}(\mathbb{R} ; X)$ of translation bounded functions; that is, for $\Psi(s) \in L_{b}^{2}(\mathbb{R} ; X)$, we have

$$
\|\Psi\|_{L_{b}^{2}(\mathbb{R} ; X)}^{2}=\sup _{t \in \mathbb{R}} \int_{t}^{t+1}\|\Psi(s)\|_{X}^{2} d s<\infty .
$$

We now give from [10] the definition and some properties of translation compact functions.

Definition 2. A function $\Psi \in L_{\text {loc }}^{2}(\mathbb{R} ; X)$ is said to be translation compact in $L_{\text {loc }}^{2}(\mathbb{R} ; X)$ if the set of its translations $\{\Psi(t+h), h \in \mathbb{R}\}$ is precompact in $L_{\text {loc }}^{2}(\mathbb{R} ; X)$ for the local convergence topology.

The set

$$
\mathscr{H}(\Psi)=[\{\Psi(t+h), h \in \mathbb{R}\}]_{L_{\text {loc }}^{2}(\mathbb{R} ; X)}
$$

is called the hull of the function $\Psi$ in the space $L_{\text {loc }}^{2}(\mathbb{R} ; X)$, where $[\cdot]_{X}$ denotes the closure in the space $X$. Note that if $\Psi$ is translation compact in $L_{\text {loc }}^{2}(\mathbb{R} ; X)$, then its hull $\mathscr{H}(\Psi)$ is compact in $L_{\text {loc }}^{2}(\mathbb{R} ; X)$. The hull $\mathscr{H}(g)$ of $g(x, t)$ in the space $L_{\text {loc }}^{2}(\mathbb{R} ; H)$ is

$$
\mathscr{H}(g)=[\{g(\cdot, t+h), h \in \mathbb{R}\}]_{L_{\mathrm{loc}}^{2}(\mathbb{R} ; H)} .
$$

The following proposition gives the existence and uniqueness of weak solutions of problems (17)-(18) (see [10] for the proof).
Proposition 3. Let $g_{0} \in L_{b}^{2}(\mathbb{R} ; H)$ and let $u_{\tau} \in H$. Problems (17)-(18) have unique solutions $u \in C\left(\mathbb{R}_{\tau} ; H\right) \cap L_{\text {loc }}^{2}\left(\mathbb{R}_{\tau} ; V\right)$ and $\partial u / \partial t \in L_{\text {loc }}^{2}\left(\mathbb{R}_{\tau} ; V^{\prime}\right)$, where $\mathbb{R}_{\tau}=[\tau,+\infty)$. The following estimates hold:

$$
\begin{aligned}
& |u(t)|^{2} \leq|u(\tau)|^{2} e^{-\lambda(t-\tau)}+\lambda^{-1}\left(1+\lambda^{-1}\right)\left\|g_{0}\right\|_{L_{b}^{2}}^{2}, \\
& |u(t)|^{2}+v \int_{\tau}^{t}\|u(s)\|^{2} d s \\
& \quad \leq|u(\tau)|+\lambda^{-1} \int_{\tau}^{t}\left|g_{0}(s)\right|^{2} d s
\end{aligned}
$$

where $\lambda=\nu \lambda_{1}$.

From Proposition 3, we can define a process $\left\{U_{g_{0}}(t, \tau)\right\}$ : $U_{g_{0}}(t, \tau) u_{\tau}=u(t), t \geq \tau$, where $u(t)$ is a solution of (17)-(18).

Now, we are given a field external force $g_{0}$ that is translation compact function in $L_{2}^{\text {loc }}(\mathbb{R} ; H)$. In particular, $g_{0}$ is translation bounded in $L_{\text {loc }}^{2}(\mathbb{R} ; H)$.

Let $\mathscr{H}\left(g_{0}\right)$ be the hull of $g_{0} \in L_{\text {loc }}^{2}(\mathbb{R} ; H)$. Consider the family of Cauchy problems

$$
\begin{aligned}
\frac{\partial u}{\partial t}+v A u+B(u, u) & =g(x, t), \\
u(\tau) & =u_{\tau}, \\
g & \in \mathscr{H}\left(g_{0}\right) .
\end{aligned}
$$

For all $g \in \mathscr{H}\left(g_{0}\right)$, problem (23) has a unique solution $u(t)$ and estimates in (22) hold. Thus the family of processes $\left\{U_{g}(t, \tau)\right\}, g \in \mathscr{H}\left(g_{0}\right)$ acting on $H$ corresponds to problem (23).

We denote by $\mathscr{K}_{g}$ the kernel of the process $\left\{\mathcal{U}_{g}^{\alpha}(t, \tau)\right\}$ with the external force $g \in \mathscr{H}\left(g_{0}\right)$. Let us recall that $\mathscr{K}_{g}$ is the family of all complete solutions $u(t), t \in \mathbb{R}$, of (23) which are bounded in the norm of $H$. The set $\mathscr{K}_{g}(s)=\{u(s), u \in$ $\left.\mathscr{K}_{g}\right\} \subset H$ is called the kernel section at $t=s$.

The following result gives the existence and the structure of the uniform attractor of the process $\left\{U_{g_{0}}(t, \tau)\right\}$ (see [10] for the proof).

Proposition 4. If $g_{0}$ is translation compact function in $L_{\text {loc }}^{2}(\mathbb{R}$; $H)$, then the process $\left\{U_{g_{0}}(t, \tau)\right\}$ corresponding to (17) with external force $g_{0}(x, s)$ has the uniform (with respect to $\tau \in$ $\mathbb{R}$ ) attractor $\mathscr{A}_{0}$ that coincides with the uniform (w.r.t $g \in$ $\mathscr{H}\left(g_{0}\right)$ ) attractor $\mathscr{A}_{\mathscr{H}\left(g_{0}\right)}$ of the family of processes $\left\{U_{g}(t, \tau)\right\}$, $g \in \mathscr{H}\left(g_{0}\right)$ and

$$
\mathscr{A}_{0}=\mathscr{A}_{\mathscr{H}\left(g_{0}\right)}=\bigcup_{g \in \mathscr{H}\left(g_{0}\right)} \mathscr{K}_{g}(0)
$$

where $\mathscr{K}_{g}$ is the kernel of the process $\left\{U_{g}(t, \tau)\right\}$. The kernel $\mathscr{K}_{g}$ is nonempty for all $g \in \mathscr{H}\left(g_{0}\right)$. 


\section{The 2D Leray- $\alpha$ Model and Its Uniform Attractor}

3.1. The 2D Leray- $\alpha$ Model. We consider the following system with periodic boundary conditions:

$$
\begin{aligned}
\frac{\partial v}{\partial t}-v \Delta v+(u \cdot \nabla) v+\nabla p & =g_{0}(x, t), \quad x \in \mathbb{T}^{2}, \\
v & =u-\alpha^{2} \Delta u, \\
\nabla \cdot u & =0, \\
\nabla \cdot v & =0 .
\end{aligned}
$$

This system is an approximation of the 2D Navier-Stokes system discussed in the previous section. The unknown functions are the vector fields $v=v(x, t)=\left(v^{1}, v^{2}\right)$ or $u=u(x, t)=\left(u^{1}, u^{2}\right)$ and the scalar function $p=p(x, t)$. In (25), $\alpha$ is a fixed positive parameter which is called the subgrid length scale of the model. For $\alpha=0$, the function $v=u$ and we obtain exactly the 2D Navier-Stokes system.

We can rewrite system (25) in an equivalent form using the standard projector $\mathscr{P}$ in $H$ and excluding the pressure as in the previous section, where all the necessary notations were defined. We obtain the system

$$
\begin{aligned}
\frac{\partial v}{\partial t}+v A v+B(u, v) & =g_{0}(x, t) \\
v & =u+\alpha^{2} A u .
\end{aligned}
$$

We supplement system (26) with the initial data

$$
v(\tau)=v_{\tau} \in H
$$

It follows from the embedding theorem in $\mathbb{R}^{2}$ that $H^{2}\left(\mathbb{T}^{2}\right) \subset$ $L^{\infty}\left(\mathbb{T}^{2}\right)$. In particular, we have the energy inequality

$$
\|u\|_{L^{\infty}\left(\mathbb{T}^{2}\right)^{2}} \leq c(\alpha)\left|u+\alpha^{2} A u\right| \leq c(\alpha)|v|,
$$

$\forall u \in H^{2} \cap V$, where $v=u+\alpha^{2} A u$ and $c(\alpha)$ is a constant that depends on $\alpha$. We obtain from inequality (28) that

$$
|B(u, v)| \leq c\|u\|_{L^{\infty}\left(\mathbb{T}^{2}\right)^{2}}\|v\| \leq c_{1}(\alpha)|v|\|v\|,
$$

where $v=u+\alpha^{2} A u$.

Consider an arbitrary function $v(\cdot) \in L_{\text {loc }}^{2}\left(\mathbb{R}_{\tau} ; V\right) \cap$ $L^{\infty}\left(\mathbb{R}_{\tau} ; H\right)$. Then, from (29), we conclude that

$$
B(u(\cdot), v(\cdot)) \in L_{\mathrm{loc}}^{2}\left(\mathbb{R}_{\tau} ; H\right) .
$$

We study weak solutions $v(x, t)$ of system (25) belonging to the space $L_{\text {loc }}^{2}\left(\mathbb{R}_{\tau} ; V\right) \cap L^{\infty}\left(\mathbb{R}_{\tau} ; H\right)$. Then

$$
\begin{aligned}
& A v \in L_{\text {loc }}^{2}\left(\mathbb{R}_{\tau} ; V^{\prime}\right), \\
& \partial_{t} v \in L_{\text {loc }}^{2}\left(\mathbb{R}_{\tau} ; V^{\prime}\right) .
\end{aligned}
$$

We now formulate the theorem on the existence and uniqueness of weak solutions of problems (26)-(27).
Theorem 5. Let $\alpha>0$, let $g_{0} \in L_{b}^{2}(\mathbb{R} ; H)$, and let $v_{\tau} \in H$. Systems (26)-(27) have unique weak solutions $v \in C\left(\mathbb{R}_{\tau} ; H\right) \cap$ $L_{\text {loc }}^{2}\left(\mathbb{R}_{\tau} ; V\right)$ and $\partial_{t} v \in L_{\text {loc }}^{2}\left(\mathbb{R}_{\tau} ; V^{\prime}\right)$. The following estimates hold:

$$
\begin{aligned}
& |u(t)|^{2} \leq|v(t)|^{2} \\
& \leq|v(\tau)|^{2} e^{-\lambda(t-\tau)}+\lambda^{-1}\left(1+\lambda^{-1}\right)\left\|g_{0}\right\|_{L_{b}^{2}(\mathbb{R} ; H)}^{2}, \\
& |v(t)|^{2}+v \int_{\tau}^{t}\|v(s)\|^{2} d s \\
& \quad \leq|v(\tau)|^{2}+\lambda^{-1} \int_{\tau}^{t}\left|g_{0}(s)\right|^{2} d s, \\
& (t-\tau)\|v(t)\|^{2} \leq C\left(t-\tau,|v(\tau)|^{2}, \int_{\tau}^{t}\left|g_{0}(s)\right|^{2} d s\right),
\end{aligned}
$$

where $\lambda=v \lambda_{1}$ and $C\left(z, R, R_{1}\right)$ is a monotone continuous function of $z=t-\tau, R$ and $R_{1}$.

To prove the estimates in (32)-(34), we will need the following lemma whose proof is given in [10].

Lemma 6. Let a real function $z(t), t \geq 0$, be uniformly continuous and satisfy the inequality

$$
\frac{d z}{d t}+\lambda z(t) \leq f(t), \quad t \geq 0
$$

where $\lambda>0, f(t) \geq 0$ for all $t \geq 0$, and $f \in L_{\text {loc }}^{1}\left(\mathbb{R}^{+}\right)$. Suppose also that

$$
\int_{t}^{t+1} f(s) d s \leq M, \quad \forall t \geq 0 .
$$

Then $z(t) \leq z(0) e^{-\lambda t}+M\left(1+\lambda^{-1}\right), \quad \forall t \geq 0$.

Proof of Theorem 5. The existence and uniqueness of weak solutions are quite analogous to the proof of the existence and uniqueness theorem for the 2D Navier-Stokes system [10]. Let us prove the estimate in (32). We take the scalar product of (26) with $v$ and use relation (16); we obtain

$$
\begin{aligned}
& \frac{1}{2} \frac{d}{d t}|v(t)|^{2}+v\|v(t)\|^{2}=\left(g_{0}(t), v(t)\right) \\
& \quad \leq \frac{v}{2}\|v(t)\|^{2}+\frac{1}{2 v}\left\|g_{0}(t)\right\|_{V^{\prime}}^{2} \\
& \quad \leq \frac{v}{2}\|v(t)\|^{2}+\frac{1}{2 v \lambda_{1}}\left|g_{0}(t)\right|^{2}
\end{aligned}
$$

Using Poincaré inequality (7), we arrive at

$$
\frac{d}{d t}|v(t)|^{2}+\lambda|v(t)|^{2} \leq \lambda^{-1}\left|g_{0}(t)\right|^{2},
$$


where $\lambda=\nu \lambda_{1}$. Applying Lemma 6 with

$$
\begin{aligned}
z(t) & =|v(t+\tau)|^{2} \\
f(t) & =\lambda^{-1}\left|g_{0}(t)\right|^{2} \\
\int_{t}^{t+1} f(s) d s & \leq \lambda^{-1} \int_{t}^{t+1}\left|g_{0}(s)\right|^{2} d s \leq \lambda^{-1}\left\|g_{0}\right\|_{L_{b}^{2}(\mathbb{R} ; H)}^{2} \\
& =M
\end{aligned}
$$

we get

$$
|v(t+\tau)|^{2} \leq|v(\tau)|^{2} e^{-\lambda t}+\lambda^{-1}\left(1+\lambda^{-1}\right)\left\|g_{0}\right\|_{L_{b}^{2}(\mathbb{R} ; H)}^{2}
$$

that is,

$$
|v(t)|^{2} \leq|v(\tau)|^{2} e^{-\lambda(t-\tau)}+\lambda^{-1}\left(1+\lambda^{-1}\right)\left\|g_{0}\right\|_{L_{b}^{2}(\mathbb{R} ; H)}^{2} .
$$

This proves (32). Multiplying (26) by $t A v$, we have

$$
\begin{gathered}
\frac{1}{2} \frac{d}{d t}\left(t\|v(t)\|^{2}\right)-\frac{1}{2}\|v(t)\|^{2}+v t|A v(t)|^{2} \\
+t(B(u, v), A v)=t\left(g_{0}(t), A v\right) .
\end{gathered}
$$

Recall that

$$
\left|\left(g_{0}(t), A v\right)\right| \leq \frac{v}{4}|A v(t)|^{2}+\frac{1}{v}\left|g_{0}(t)\right|^{2}
$$

From (29), we have

$$
\begin{aligned}
|(B(u, v), A v)| & \leq|B(u, v)||A v| \leq c_{1}(\alpha)|v|\|v\||A v| \\
& \leq \frac{v}{4}|A v(t)|^{2}+\frac{c_{1}^{2}(\alpha)}{v}|v|^{2}\|v\|^{2} .
\end{aligned}
$$

Replacing (43) and (44) in (42), we get

$$
\begin{aligned}
& \frac{d}{d t}\left\{t\|v(t)\|^{2}\right\}+v t|A v(t)|^{2} \\
& \quad \leq\|v(t)\|^{2}+\frac{2 t}{v}\left|g_{0}(t)\right|^{2}+\frac{2 c_{1}^{2}(\alpha)}{v} t|v(t)|^{2}\|v(t)\|^{2}
\end{aligned}
$$

Let us set $y(t)=t\|v(t)\|^{2}$ and obtain

$$
\frac{d y}{d t} \leq \frac{2 c_{1}^{2}(\alpha)}{v}|v(t)|^{2} y+\|v(t)\|^{2}+\frac{2 t}{v}\left|g_{0}(t)\right|^{2} \text {. }
$$

Using Gronwall's lemma, we obtain

$$
\begin{aligned}
t\|v(t)\|^{2} \leq & \left(\int_{0}^{t}\left(\|v(s)\|^{2}+s \frac{2}{v}\left|g_{0}(s)\right|^{2}\right) d s\right) \\
& \cdot \exp \left(\int_{0}^{t} \frac{2 c_{1}^{2}(\alpha)}{v}|v(s)|^{2} d s\right) .
\end{aligned}
$$

From the estimate in (33), we deduce from (47) that

$$
\begin{aligned}
& t\|v(t)\|^{2} \leq \frac{1}{v}\left(|v(0)|^{2}+\left(\lambda^{-1}+2 t\right) \int_{0}^{t}\left|g_{0}(s)\right|^{2} d s\right) \\
& \cdot \exp \left(\frac{2 c_{1}^{2}(\alpha)}{v^{2}}|v(0)|^{2}\right. \\
& \left.+\frac{2 c_{1}^{2}(\alpha) \lambda^{-1}}{v^{2}} \int_{0}^{t}\left|g_{0}(s)\right|^{2} d s\right) \leq C\left(t,|v(0)|^{2},\right. \\
& \left.\int_{0}^{t}\left|g_{0}(s)\right|^{2} d s\right),
\end{aligned}
$$

where

$$
\begin{aligned}
C\left(z, R, R_{1}\right)= & \frac{1}{v}\left(R+\left(\lambda^{-1}+2 z\right) R_{1}\right) \\
& \cdot \exp \left(\frac{2 c_{1}^{2}(\alpha)}{v^{2}} R+\frac{2 c_{1}^{2}(\alpha) \lambda^{-1}}{v^{2}} R_{1}\right) .
\end{aligned}
$$

This ends the proof of Theorem 5 .

Remark 7. We note that the estimates in (32) and (33) are independent of $\alpha$. This fact plays the key role in the proof of the convergence of solutions of the $2 \mathrm{D}$ Leray- $\alpha$ model to the solution of the 2D Navier-Stokes system as $\alpha \rightarrow 0^{+}$.

3.2. The Uniform Attractor $\mathscr{A}^{\alpha}$ of the $2 D$ Leray- $\alpha$ Model. In this subsection, we prove the existence of the uniform attractor for the 2D Leray- $\alpha$ model. We consider the process $\left\{\mathcal{U}_{g_{0}}^{\alpha}(t, \tau)\right\}, t \geq \tau, \tau \in \mathbb{R}$ corresponding to problems (26)(27). More precisely, the mapping $\mathscr{U}_{g_{0}}^{\alpha}(t, \tau): H \rightarrow H$ is defined by

$$
\mathcal{U}_{g_{0}}^{\alpha}(t, \tau) v_{\tau}=v(t)
$$

for all $v_{\tau} \in H, t \geq \tau, \tau \in \mathbb{R}$, where $v$ is solution of (26)(27). It follows from (32) that the process $\left\{\mathcal{U}_{g_{0}}^{\alpha}(t, \tau)\right\}$ has the uniform (w.r.t. $\tau \in \mathbb{R}$ ) absorbing set

$$
B_{0}=\left\{v \in H:|v|^{2} \leq 2 R_{0}^{2}\right\},
$$

where $R_{0}^{2}=\lambda^{-1}\left(1+\lambda^{-1}\right)\left\|g_{0}\right\|_{L_{b}^{2}(\mathbb{R} ; H)}^{2}$ and the set $B_{0}$ is bounded in $H$. Therefore, for any bounded (in $H$ ) set $\mathcal{O}$, there exists a time $t(\mathcal{O})$ such that

$$
\mathcal{U}_{g_{0}}^{\alpha}(t+\tau, \tau) \mathcal{O} \subset B_{0},
$$

for all $t>t(\mathcal{O})$ and $\tau \in \mathbb{R}$.

Proposition 8. The process $\left\{\mathcal{U}_{g_{0}}^{\alpha}(t, \tau)\right\}$ associated with (26)(27) is uniformly compact in $H$ and has a uniformly absorbing set $B_{1}$ (bounded in $V$ ) defined by

$$
B_{1}=\bigcup_{\tau \in \mathbb{R}} \mathcal{U}_{g_{0}}^{\alpha}(\tau+1, \tau) B_{0}
$$

where $B_{0}$ is given by (51). Moreover, the process $\left\{\mathcal{U}_{g_{0}}^{\alpha}(t, \tau)\right\}$ has a uniform attractor $\mathscr{A}^{\alpha}$ which satisfies

$$
\mathscr{A}^{\alpha} \subset B_{0} \cup B_{1} \text {. }
$$


Proof. From (34) and (51), it is clear that $B_{1}$ is bounded in $V$ and hence is relatively compact in $H$. From (34), it is also clear that $B_{1}$ is uniform (with respect to $\tau \in \mathbb{R}$ ) absorbing set for the process $\left\{\mathcal{U}_{g_{0}}^{\alpha}(t, \tau)\right\}$. The rest of the proof of the proposition follows the general theory on uniform global attractors [10]. This ends the proof of the proposition.

From the general theory on uniform global attractors in [10], the global attractor $\mathscr{A}^{\alpha}$ given in Proposition 8 satisfies the following:

(i) For any bounded (in $H)$ set $\mathcal{O}, \sup _{\tau \in \mathbb{R}} \operatorname{dist}_{H}\left(\mathcal{U}_{g_{0}}^{\alpha}(t+\right.$ $\left.\tau, \tau) \mathcal{O}, \mathscr{A}^{\alpha}\right) \rightarrow 0$ as $t \rightarrow \infty$.

(ii) $\mathscr{A}^{\alpha}$ is the minimal set that satisfies (i).

3.3. The Structure of the Uniform Attractor of the $2 D$ Leray- $\alpha$ Model. We consider the system

$$
\begin{aligned}
\frac{\partial v}{\partial t}+v A v+B(u, v) & =g_{0}, \\
v(\tau) & =v_{\tau}, \\
v & =u+\alpha^{2} A u .
\end{aligned}
$$

We assume that $g_{0}$ is translation compact in the space $L_{\text {loc }}^{2}(\mathbb{R} ; H)$. Let $\mathscr{H}\left(g_{0}\right)$ be the hull of $g_{0}$ in $L_{\text {loc }}^{2}(\mathbb{R} ; H)$. For all $g \in \mathscr{H}\left(g_{0}\right)$, the problem

$$
\begin{aligned}
\frac{\partial v}{\partial t}+v A v+B(u, v) & =g(t, x) \\
v & =u+\alpha^{2} A u \\
v(\tau) & =v_{\tau}
\end{aligned}
$$

has a unique solution $v(t)$ and the estimates in (32)-(34) hold. For $g \in \mathscr{H}\left(g_{0}\right)$, system (56) generates a process $\left\{\mathscr{U}_{g}^{\alpha}(t, \tau)\right\}$ that satisfies the same properties as the process $\left\{\mathcal{U}_{g_{0}}^{\alpha}(t, \tau)\right\}$. The family of processes $\left\{\mathcal{U}_{g}^{\alpha}(t, \tau)\right\}, g \in \mathscr{H}(g)$, acting on $H$ corresponds to (56).

Proposition 9. The family of processes $\left\{\mathcal{U}_{g}^{\alpha}(t, \tau)\right\}, g \in$ $\mathscr{H}\left(g_{0}\right)$, corresponding to (56) is uniformly (with respect to $g \in$ $\mathscr{H}\left(g_{0}\right)$ ) bounded, uniformly compact, and $\left(H \times \mathscr{H}\left(g_{0}\right), H\right)$ continuous.

Proof. The uniform boundedness of the family of processes $\left\{\mathcal{U}_{g}^{\alpha}(t, \tau)\right\}, g \in \mathscr{H}\left(g_{0}\right)$, follows from (32) and the fact that

$$
\|g\|_{L_{b}^{2}(\mathbb{R} ; H)}^{2} \leq\left\|g_{0}\right\|_{L_{b}^{2}(\mathbb{R} ; H)}^{2}, \quad \forall g \in \mathscr{H}\left(g_{0}\right)
$$

This estimate also implies that the set $B_{0}=\left\{v \in H ;|v|^{2} \leq\right.$ $\left.2 R_{0}^{2}\right\}$, where $R_{0}^{2}=\lambda^{-1}\left(1+\lambda^{-1}\right)\left\|g_{0}\right\|_{L_{b}^{2}(\mathbb{R} ; H)}^{2}$, is uniformly (with respect to $g \in \mathscr{H}\left(g_{0}\right)$ absorbing. The set

$$
B_{1}=\bigcup_{g \in \mathscr{H}\left(g_{0}\right)} \bigcup_{\tau \in \mathbb{R}} \mathcal{u}_{g}(\tau+1, \tau) B_{0}
$$

is also uniformly absorbing. By (34), the set $B_{1}$ is bounded in $V$ and therefore, by the compactness of the embedding $V \hookrightarrow$ $H, B_{1}$ is precompact in $H$. Hence the family $\left\{\mathcal{U}_{g}^{\alpha}(t, \tau)\right\}, g \in$ $\mathscr{H}\left(g_{0}\right)$, is uniformly compact.

Let us verify the $\left(H \times \mathscr{H}\left(g_{0}\right), H\right)$-continuity of the processes $\left\{\mathcal{U}_{g}^{\alpha}(t, \tau)\right\}, g \in \mathscr{H}\left(g_{0}\right)$. We consider two symbols $g_{1}$ and $g_{2}$ and the corresponding solutions $v_{1}$ and $v_{2}$ of problem (56) with initial data $v_{1 \tau}$ and $v_{2 \tau}$, respectively. Denote

$$
\begin{aligned}
w(t) & =v_{1}(t)-v_{2}(t)=\mathscr{U}_{g_{1}}(t, \tau) v_{1 \tau}-\mathcal{U}_{g_{2}}(t, \tau) v_{2 \tau}, \\
q & =g_{1}-g_{2} .
\end{aligned}
$$

The function $w$ satisfies the equation

$$
\frac{\partial w}{\partial t}+v A w+B\left(u_{1}, v_{1}\right)-B\left(u_{2}, v_{2}\right)=q .
$$

We take the inner product of (60) with $w$; we obtain

$$
\frac{1}{2} \frac{d}{d t}|w|^{2}+v\|w\|^{2}+\left\langle B\left(u_{1}-u_{2}, v_{2}\right), w\right\rangle=(q, w)
$$

Using the estimate in (10), we arrive at

$$
\begin{aligned}
& \left|\left\langle B\left(u_{1}-u_{2}, v_{2}\right), w\right\rangle\right| \\
& \quad \leq c\left|u_{1}-u_{2}\right|^{1 / 2}\left\|u_{1}-u_{2}\right\|^{1 / 2}\left\|v_{2}\right\||w|^{1 / 2}\|w\|^{1 / 2} \\
& \quad \leq c|w|^{1 / 2}|w|^{1 / 2}\|w\|^{1 / 2}\|w\|^{1 / 2}\left\|v_{2}\right\| \\
& \quad \leq c|w|\|w\|\left\|v_{2}\right\| \leq \frac{v}{4}\|w\|^{2}+c|w|^{2}\left\|v_{2}\right\|^{2} .
\end{aligned}
$$

Also we have

$$
(q, w) \leq|q||w| \leq \sqrt{\lambda^{-1}}|q|\|w\| \leq \frac{\nu}{4}\|w\|^{2}+c_{1}|q|^{2} .
$$

Using (62) and (63) in (61), we get

$$
\frac{d}{d t}|w|^{2}+v\|w\|^{2} \leq c|w|^{2}\left\|v_{2}\right\|^{2}+c_{1}|q|^{2} .
$$

Let us set $y(t)=|w(t)|^{2}$ and we obtain

$$
\frac{d}{d t} y(t) \leq c\left\|v_{2}\right\|^{2} y(t)+c_{1}|q|^{2}
$$

Using Gronwall's lemma, we obtain

$$
\begin{aligned}
|w(t)|^{2} \leq & \left(|w(\tau)|^{2}+\int_{\tau}^{t} c_{1}|q(s)|^{2} d s\right) \\
& \cdot \exp \left(\int_{\tau}^{t} c\left\|v_{2}(s)\right\|^{2} d s\right)
\end{aligned}
$$

With the estimate in (33), we get

$$
\int_{\tau}^{t}\left\|v_{2}(s)\right\|^{2} d s \leq \frac{1}{v}\left(\left|v_{2}(\tau)\right|^{2}+\lambda^{-1} \int_{\tau}^{t}\left|g_{2}(s)\right|^{2} d s\right) .
$$

The estimate in (67) proves that $\int_{\tau}^{t}\left\|v_{2}(s)\right\|^{2} d s$ is bounded, and (66) implies the $\left(H \times \mathscr{H}\left(g_{0}\right), H\right)$-continuity of the family of processes $\left\{\mathcal{U}_{g}^{\alpha}(t, \tau)\right\}, g \in \mathscr{H}\left(g_{0}\right)$. This ends the proof of the proposition. 
Theorem 10. If $g_{0}$ is translation compact in $L_{2}^{\text {loc }}(\mathbb{R} ; H)$, then the process $\left\{\mathcal{U}_{g_{0}}(t, \tau)\right\}$ corresponding to (55) with external force $g_{0}(x, t)$ has the uniform (with respect to $\tau \in \mathbb{R}$ ) attractor $\mathscr{A}^{\alpha}$ that coincides with the uniform (with respect to $g \in \mathscr{H}\left(g_{0}\right)$ ) attractor $\mathscr{A}_{\mathscr{H}\left(g_{0}\right)}^{\alpha}$ of the family of processes $\left\{\mathcal{U}_{g}^{\alpha}(t, \tau)\right\}, g \in$ $\mathscr{H}\left(g_{0}\right)$.

Moreover,

$$
\mathscr{A}^{\alpha}=\mathscr{A}_{\mathscr{H}\left(g_{0}\right)}^{\alpha}=\bigcup_{g \in \mathscr{H}\left(g_{0}\right)} \mathscr{K}_{g}^{\alpha}(0),
$$

where $\mathscr{K}_{g}^{\alpha}$ is the kernel of the process $\left\{\mathcal{U}_{g}^{\alpha}(t, \tau)\right\}$. The kernel $\mathscr{K}_{g}^{\alpha}$ is nonempty for all $g \in \mathscr{H}\left(g_{0}\right)$.

In the next section, we study the asymptotic behavior of the uniform attractor of the $2 \mathrm{D}$ Leray- $\alpha$ model.

\section{Convergence of the Uniform Attractors of the 2D Leray- $\alpha$ Model}

In the previous sections, we have proven the existence and the structure of the uniform attractor:

(a) $\mathscr{A}^{\alpha}$ of the process $\left\{\mathcal{U}_{g_{0}}^{\alpha}(t, \tau)\right\}$ generated by the solutions of the 2D Leray- $\alpha$ model.

(b) $\mathscr{A}_{0}$ of the process $\left\{\mathcal{U}_{g_{0}}(t, \tau)\right\}$ generated by the solutions of the 2D Navier-Stokes system.

Our aim in this section is to prove the convergence of the uniform attractors $\mathscr{A}^{\alpha}$ to the uniform attractor $\mathscr{A}_{0}$ as $\alpha$ approaches 0 ; that is,

$$
\lim _{n \rightarrow \infty} \operatorname{dist}_{H}\left(\mathscr{A}^{\alpha_{n}}, \mathscr{A}_{0}\right)=0,
$$

$$
\text { if } \alpha_{n} \rightarrow 0^{+} \text {. }
$$

The following proposition is the key.

Proposition 11. Let $\left\{g_{n}\right\}, g \in \mathscr{H}\left(g_{0}\right)$, and a sequence of functions $v_{\alpha_{n}}(t) \in \mathscr{K}_{g_{n}}^{\alpha_{n}}(t)$ satisfy the following conditions:

(1) $\alpha_{n} \rightarrow 0^{+}$as $n \rightarrow \infty$.

(2) $g_{n} \rightarrow g$ in $\mathscr{H}\left(g_{0}\right)$ as $n \rightarrow \infty$.

(3) $v_{\alpha_{n}}(t) \rightarrow v(t)$ in $H$ as $n \rightarrow \infty$.

Then $v$ is a weak solution of the 2D Navier-Stokes system with external force $g$; that is, $v \in \mathscr{K}_{g}$.

For the proof of this proposition, we need an estimate for the derivative $\partial_{t} v$ in which constants are independent of $\alpha$ similar to that proven for $v$ in (32)-(33).

Proposition 12. Let $g_{0} \in L_{b}^{2}(\mathbb{R} ; H)$ and let $v_{\tau} \in H$. Then any solution $v(t)$ of (26)-(27) satisfies the following inequalities:

$$
\begin{aligned}
& \left(\int_{\tau}^{T}\left\|\partial_{t} v(s)\right\|_{V^{*}}^{4 / 3} d s\right)^{3 / 4} \leq c\left|v_{\tau}\right|^{2}+R_{2}^{2}, \\
& \left(\int_{\tau}^{T}\left\|\partial_{t} v(s)\right\|_{V^{*}}^{2} d s\right)^{1 / 2} \leq c\left|v_{\tau}\right|^{2}+R_{2}^{2},
\end{aligned}
$$

where $c$ depends on $\lambda_{1}, v . R_{2}$ depends on $\lambda_{1}$, v and $\left\|g_{0}\right\|_{L_{b}^{2}(\mathbb{R} ; H)}$. The numbers $c$ and $R_{2}$ are independent of $\alpha$.

Proof. Consider the operator $B(u(t), v(t))$, where $v=u+$ $\alpha^{2} A u$. We note that

$$
\begin{gathered}
|u| \leq|v|, \\
\|u\| \leq\|v\| .
\end{gathered}
$$

From inequalities (10) and (72), we get

$$
\|B(u, v)\|_{V^{*}} \leq c|u|^{1 / 2}\|u\|^{1 / 2}\|v\| \leq c|v|^{1 / 2}\|v\|^{3 / 2}
$$

We deduce that

$$
\begin{aligned}
& \left(\int_{\tau}^{T}\|B(u(s), v(s))\|_{V^{*}}^{4 / 3} d s\right)^{3 / 4} \\
& \quad \leq c\left(\int_{\tau}^{T}|v(s)|^{2 / 3}\|v(s)\|^{2} d s\right)^{3 / 4} \leq c \\
& \cdot \operatorname{ess} \sup _{s \in[\tau, T]}|v(s)|^{1 / 2}\left(\int_{\tau}^{T}\|v(s)\|^{2} d s\right)^{3 / 4} \\
& \quad \leq c\left(|v(\tau)|^{2} e^{-\lambda T}+\lambda^{-1}\left(1+\lambda^{-1}\right)\left\|g_{0}\right\|_{L_{b}^{2}(\mathbb{R} ; H)}^{2}\right)^{1 / 4} \\
& \quad \cdot\left(\frac{1}{v}|v(\tau)|^{2}+\frac{\lambda^{-1}}{v} \int_{\tau}^{T}\left|g_{0}(s)\right|^{2} d s\right)^{3 / 4} \\
& \quad \leq c\left(|v(\tau)|^{2} e^{-\lambda T}+\lambda^{-1}\left(1+\lambda^{-1}\right)\left\|g_{0}\right\|_{L_{b}^{2}(\mathbb{R} ; H)}^{2}\right)^{1 / 4} \\
& \quad \cdot\left(\frac{1}{v}|v(\tau)|^{2}+\frac{\lambda^{-1}}{v}(T+1)\left\|g_{0}\right\|_{L_{b}^{2}(\mathbb{R} ; H)}^{2}\right)^{3 / 4} \\
& \quad \leq c\left(|v(\tau)|^{2}+\lambda^{-1}\left(1+\lambda^{-1}\right)\left\|g_{0}\right\|_{L_{b}^{2}(\mathbb{R} ; H)}^{2}\right. \\
& \quad+\lambda^{-1}(T+1)\left\|g_{0}\right\|_{L_{b}^{2}(\mathbb{R} ; H)}^{2} \leq c|v(\tau)|^{2}+\left(R_{2}^{\prime}\right)^{2}
\end{aligned}
$$

where $\left(R_{2}^{\prime}\right)^{2}=c \lambda^{-1}\left(1+\lambda^{-1}\right)\left\|g_{0}\right\|_{L_{b}^{2}(\mathbb{R} ; H)}^{2}+\lambda^{-1}(T+1)\left\|g_{0}\right\|_{L_{b}^{2}(\mathbb{R} ; H)}^{2}$. Using the triangle inequality, it follows from (26) that

$$
\begin{aligned}
& \left(\int_{\tau}^{T}\left\|\partial_{t} v(s)\right\|_{V^{*}}^{4 / 3} d s\right)^{3 / 4} \\
& \leq \nu\left(\int_{\tau}^{T}\|A v(s)\|_{V^{*}}^{4 / 3} d s\right)^{3 / 4} \\
& \quad+\left(\int_{\tau}^{T}\|B(u(s), v(s))\|_{V^{*}}^{4 / 3} d s\right)^{3 / 4} \\
& \quad+\left(\int_{\tau}^{T}\left\|g_{0}(s)\right\|_{V^{*}}^{4 / 3} d s\right)^{3 / 4}
\end{aligned}
$$




$$
\begin{aligned}
\leq & \nu\left(\int_{\tau}^{T}\|v(s)\|^{4 / 3} d s\right)^{3 / 4} \\
& +\left(\int_{\tau}^{T}\|B(u(s), v(s))\|_{V^{*}}^{4 / 3} d s\right)^{3 / 4} \\
& +\lambda^{-1 / 2}\left(\int_{\tau}^{T}\left|g_{0}(s)\right|^{4 / 3} d s\right)^{3 / 4} \\
\leq & \nu\left(\int_{\tau}^{T}\|v(s)\|^{2} d s\right)^{1 / 2} \\
& +\left(\int_{\tau}^{T}\|B(u(s), v(s))\|_{V^{*}}^{4 / 3} d s\right)^{3 / 4} \\
& +(T+1) \lambda^{-1 / 2}\left\|g_{0}\right\|_{L_{b}^{2}(\mathbb{R} ; H)}+1 \leq c|v(\tau)|^{2}+R_{2}^{2} \\
& +\lambda^{-1 / 2}\left(\int_{\tau}^{T}\left|g_{0}(s)\right|^{2} d s\right)^{1 / 2} \\
& +(T+1) \lambda^{-f r a c ~ 12}\left\|g_{0}\right\|_{L_{b}^{2}((R) ; H)} \\
\leq & v\left(\frac{1}{v}|v(\tau)|^{2}+\frac{\lambda^{-1}}{v} \int_{\tau}^{T}\left|g_{0}(s)\right|^{2} d s\right)^{1 / 2} \\
& +\lambda^{-1}(T+1)\left\|g_{0}\right\|_{L_{b}^{2}(\mathbb{R} ; H)}^{2}\left(R_{2}^{\prime}\right)^{2}
\end{aligned}
$$

where $R_{2}^{2}=\lambda^{-1}(T+1)\left\|g_{0}\right\|_{L_{b}^{2}(\mathbb{R} ; H)}^{2}+\left(R_{2}^{\prime}\right)^{2}+(T+1) \lambda^{-1 / 2}$ $\left\|g_{0}\right\|_{L_{b}^{2}(\mathbb{R} ; H)}+1$. This proves $(70)$.

For the proof of (71), we use inequalities (11) and (72) and we get

$$
\begin{aligned}
\|B(u, v)\|_{V^{*}} & \leq c|u|^{1 / 2}\|u\|^{1 / 2}|v|^{1 / 2}\|v\|^{1 / 2} \\
& \leq|v|^{1 / 2}\|v\|^{1 / 2}|v|^{1 / 2}\|v\|^{1 / 2} \leq c|v|\|v\| .
\end{aligned}
$$

We then have

$$
\begin{aligned}
& \left(\int_{\tau}^{T}\|B(u(s), v(s))\|_{V^{*}}^{2} d s\right)^{1 / 2} \\
& \quad \leq c\left(\int_{\tau}^{T}|v(s)|^{2}\|v(s)\|^{2} d s\right)^{1 / 2} \leq c \\
& \cdot \operatorname{ess} \sup _{s \in[\tau, T]}|v(s)|\left(\int_{\tau}^{T}\|v(s)\|^{2} d s\right)^{1 / 2}
\end{aligned}
$$

$$
\begin{aligned}
& \leq c\left(|v(\tau)|^{2} e^{-\lambda T}+\lambda^{-1}\left(1+\lambda^{-1}\right)\left\|g_{0}\right\|_{L_{2}^{b}(\mathbb{R} ; H)}^{2}\right)^{1 / 2} \\
& \cdot\left(\frac{1}{v}|v(\tau)|^{2}+\frac{\lambda^{-1}}{v} \int_{\tau}^{T}\left|g_{0}(s)\right|^{2} d s\right)^{1 / 2} \\
& \leq c\left(|v(\tau)|^{2} e^{-\lambda T}+\lambda^{-1}\left(1+\lambda^{-1}\right)\left\|g_{0}\right\|_{L_{2}^{b}(\mathbb{R} ; H)}^{2}\right)^{1 / 2} \\
& \cdot\left(\frac{1}{v}|v(\tau)|^{2}+\frac{\lambda^{-1}}{v}(T+1)\left\|g_{0}\right\|_{L_{b}^{2}(\mathbb{R} ; H)}^{2}\right)^{1 / 2} \\
& \leq c\left(|v(\tau)|^{2}+\lambda^{-1}\left(1+\lambda^{-1}\right)\left\|g_{0}\right\|_{L_{b}^{2}(\mathbb{R} ; H)}^{2}\right. \\
& \left.+\lambda^{-1}(T+1)\left\|g_{0}\right\|_{L_{b}^{2}(\mathbb{R} ; H)}^{2}\right) \leq c|v(\tau)|^{2}+\left(R_{2}^{\prime}\right)^{2} .
\end{aligned}
$$

It follows from (26) that

$$
\begin{aligned}
& \left(\int_{\tau}^{T}\left\|\partial_{t} v(s)\right\|_{V^{*}}^{2} d s\right)^{1 / 2} \\
& \leq v\left(\int_{\tau}^{T}\|A v(s)\|_{V^{*}}^{2} d s\right)^{1 / 2} \\
& +\left(\int_{\tau}^{T}\|B(u(s), v(s))\|_{V^{*}}^{2} d s\right)^{1 / 2} \\
& +\left(\int_{\tau}^{T}\left\|g_{0}(s)\right\|_{V^{*}}^{2} d s\right)^{1 / 2} \\
& \leq v\left(\int_{\tau}^{T}\|v(s)\|^{2} d s\right)^{1 / 2} \\
& +\left(\int_{\tau}^{T}\|B(u(s), v(s))\|_{V^{*}}^{2} d s\right)^{1 / 2} \\
& +\lambda^{-1 / 2}\left(\int_{\tau}^{T}\left|g_{0}(s)\right|^{2} d s\right)^{1 / 2} \\
& \leq v\left(\int_{\tau}^{T}\|v(s)\|^{2} d s\right)^{1 / 2} \\
& +\left(\int_{\tau}^{T}\|B(u(s), v(s))\|_{V^{*}}^{2} d s\right)^{1 / 2} \\
& +\lambda^{-1 / 2}\left(\int_{\tau}^{T}\left|g_{0}(s)\right|^{2} d s\right)^{1 / 2} \\
& \leq \nu\left(\frac{1}{\nu}|v(\tau)|^{2}+\frac{\lambda^{-1}}{\nu} \int_{\tau}^{T}\left|g_{0}(s)\right|^{2} d s\right)^{1 / 2} \\
& +c|v(\tau)|^{2}+\left(R_{2}^{\prime}\right)^{2}+(T+1) \lambda^{-1 / 2}\left\|g_{0}\right\|_{L_{b}^{2}((R) ; H)} \\
& \leq c|v(\tau)|^{2}+\lambda^{-1}(T+1)\left\|g_{0}\right\|_{L_{b}^{2}(\mathbb{R} ; H)}^{2}+\left(R_{2}^{\prime}\right)^{2} \\
& +(T+1) \lambda^{-1 / 2}\left\|g_{0}\right\|_{L_{b}^{2}(\mathbb{R} ; H)}+1 \leq c|v(\tau)|^{2}+R_{2}^{2} .
\end{aligned}
$$

This ends the proof of the proposition. 
Proof of Proposition 11. We prove that $v$ is a weak solution of the 2D Navier-Stokes system on every interval $(\tau, T)$. The function $v_{\alpha_{n}}$ satisfies the equation

$$
\partial_{t} v_{\alpha_{n}}+v A v_{\alpha_{n}}+B\left(u_{\alpha_{n}}, v_{\alpha_{n}}\right)=g_{n}
$$

From the estimates in (32)-(33) and (71), we have

$$
\begin{aligned}
& \left|v_{\alpha_{n}}(t)\right|^{2} \\
& \leq|v(\tau)|^{2} e^{-\lambda(t-\tau)}+\lambda^{-1}\left(1+\lambda^{-1}\right)\left\|g_{n}\right\|_{L_{b}^{2}(\mathbb{R} ; H)}^{2}, \\
& v \int_{\tau}^{t}\left\|v_{\alpha_{n}}(s)\right\|^{2} d s \leq|v(\tau)|^{2}+\lambda^{-1} \int_{\tau}^{t}\left|g_{n}(s)\right|^{2} d s, \\
& \left(\int_{\tau}^{T}\left\|\partial_{t} v_{\alpha_{n}}(s)\right\|_{V^{*}}^{2} d s\right)^{1 / 2} \\
& \leq c|v(\tau)|^{2}+2 \lambda^{-1}(T+1)\left\|g_{n}\right\|_{L_{b}^{2}(\mathbb{R} ; H)}^{2} \\
& \quad+c \lambda^{-1}\left(1+\lambda^{-1}\right)\left\|g_{n}\right\|_{L_{b}^{2}(\mathbb{R} ; H)}^{2} \\
& +(T+1) \lambda^{-1 / 2}\left\|g_{n}\right\|_{L_{b}^{2}(\mathbb{R} ; H)}+1 .
\end{aligned}
$$

Since each bounded sequence in a reflexive Banach space has a weakly convergent subsequence (see [20], Theorem 21.D, p. 255), we can choose a subsequence $\left\{v_{\alpha_{n}}(t)\right\}$ of $\left\{v_{\alpha_{n}}(t)\right\}$ such that

$$
\begin{gathered}
v_{\alpha_{n}}(t) \rightarrow v(t) \quad \text { in } H, \\
\frac{\partial v_{\alpha_{n}}}{\partial t} \rightarrow v^{\prime}(t) \quad \text { in } L^{2}\left(\tau, T ; V^{\prime}\right), \\
v_{\alpha_{n}} \rightarrow v \quad \text { in } L^{2}(\tau, T ; V),
\end{gathered}
$$

as $n \rightarrow \infty$. The convergence (82) uses the fact that the generalized derivatives are compatible with the weak limits (see [20], Proposition 23.19, p. 419). From (83), we obtain

$$
A v_{\alpha_{n}} \rightarrow A v \text { in } L^{2}\left(\tau, T ; V^{\prime}\right) .
$$

In order to establish the equality, it is sufficient to prove that the sequence $B\left(u_{\alpha_{n}}, v_{\alpha_{n}}\right)$ converges to $B(v(\cdot), v(\cdot))$ in $\mathscr{D}\left(\tau, T ; V^{\prime}\right)$ as $n \rightarrow \infty$. Notice that

$$
u_{\alpha_{n}} \rightarrow v \quad \text { weakly in } L^{2}(\tau, T ; V) .
$$

Indeed, the function $u_{\alpha_{n}}$ satisfies the equation

$$
u_{\alpha_{n}}+\alpha_{n}^{2} A u_{\alpha_{n}}=v_{\alpha_{n}} \text {. }
$$

Since $u_{\alpha_{n}}$ is bounded in $L^{2}(\tau, T ; V)$, then, passing to a subsequence, we may assume that $u_{\alpha_{n}}$ converges to a function $w(\cdot)$ weakly in $L^{2}(\tau, T ; V)$; that is,

$$
u_{\alpha_{n}} \rightarrow w \text { in } L^{2}(\tau, T ; V)
$$

Then the sequence $A u_{\alpha_{n}} \rightarrow A w$ weakly in $L^{2}\left(\tau, T ; V^{\prime}\right)$ and

$$
\alpha_{n} A u_{\alpha_{n}} \rightarrow 0 \quad \text { weakly in } L^{2}\left(\tau, T: V^{\prime}\right) \text {. }
$$

Therefore, in equality (86), we may pass to the limit in the space $L^{2}\left(\tau, T: V^{\prime}\right)$ and obtain that

$$
w=\lim _{n \rightarrow \infty} u_{\alpha_{n}}=\lim _{n \rightarrow \infty} v_{\alpha_{n}}=v .
$$

Then, (87) and (89) imply (85).

From (71), the sequences $\partial_{t} v_{n}$ and $\partial_{t} u_{n}$ are bounded in $L^{2}\left(\tau, T ; V^{\prime}\right)$. Then the Aubin compactness theorem [21] implies that, passing to a subsequence, we may assume that $v_{\alpha_{n}}$ and $u_{\alpha_{n}}$ converge to $v(\cdot)$ strongly in $L^{2}(\tau, T ; H)$. Therefore, we may assume that

$$
\begin{aligned}
& \left.v_{\alpha_{n}}(x, t) \longrightarrow v(x, t) \quad \text { for a.e. }(x, t) \in \mathbb{T}^{2} \times\right] \tau, T[, \\
& \left.u_{\alpha_{n}}(x, t) \longrightarrow v(x, t) \quad \text { for a.e. }(x, t) \in \mathbb{T}^{2} \times\right] \tau, T[.
\end{aligned}
$$

We recall that

$$
B\left(u_{\alpha_{n}}, v_{\alpha_{n}}\right)=\mathscr{P} \sum_{i=1}^{2} \partial_{i}\left(u_{\alpha_{n}}^{i} v_{\alpha_{n}}\right) .
$$

It follows from (90) that

$$
\begin{aligned}
& u_{\alpha_{n}}^{i}(x, t) v_{\alpha_{n}}(x, t) \longrightarrow v^{i}(x, t) v(x, t) \\
& \left.\quad \text { for a.e. }(x, t) \in \mathbb{T}^{2} \times\right] \tau, T[
\end{aligned}
$$

Using the estimate in (11), we deduce that

$$
u_{\alpha_{n}}^{i} v_{\alpha_{n}} \text { is bounded in } L^{2}(\tau, T ; H), L^{2}\left(\mathbb{T}^{2} \times\right] \tau, T[)^{2} \text {. }
$$

Applying the known lemma on weak convergence from [21], we conclude from (92) and (93) that

$$
u_{\alpha_{n}}^{i} v_{\alpha_{n}} \rightarrow v^{i} v
$$

weakly in $L^{2}\left(\mathbb{T}^{2} \times\right] \tau, T[)^{2}$ and weakly in $L^{2}(\tau, T ; H)$. We then deduce from (91) that

$$
B\left(u_{\alpha_{n}}, v_{\alpha_{n}}\right) \rightarrow B(v, v) \quad \text { weakly in } L^{2}\left(\tau, T ; V^{\prime}\right) .
$$

We have then proven that $v(\cdot)$ is a weak solution of the $2 \mathrm{D}$ Navier-Stokes equations with external force $g$. This completes the proof of the proposition.

Now we present and prove the main result of this paper.

Theorem 13. Let $\mathscr{A}^{\alpha_{n}}$ be the uniform attractor of the 2D Leray$\alpha$ model and let $\mathscr{A}_{0}$ be the uniform attractor of the $2 D$ NavierStokes system. Then one has

$$
\mathscr{A}^{\alpha_{n}} \text { converges to } \mathscr{A}_{0} \text { as } n \text { approaches } \infty \text {; }
$$

that is,

$$
\lim _{n \rightarrow \infty} \operatorname{dist}_{H}\left(\mathscr{A}^{\alpha_{n}}, \mathscr{A}_{0}\right)=0
$$


Remark 14. In (97), dist $_{H}$ denotes the Hausdorff semidistance defined by

$$
\operatorname{dist}_{H}(X, Y)=\sup _{x \in X} \inf _{y \in Y}|x-y| \text {. }
$$

Proof of Theorem 13. Assume that $\operatorname{dist}_{H}\left(\mathscr{A}^{\alpha_{n}}, \mathscr{A}_{0}\right) \nrightarrow 0$. Hence, by the compactness of $\mathscr{A}_{0}$, we can choose a positive constant $\delta>0$ and a subsequence $\{m\}$ of $\{n\}$ and $\psi_{m} \in \mathscr{A}^{\alpha_{m}}$ satisfying

$$
\operatorname{dist}_{H}\left(\psi_{m}, \mathscr{A}_{0}\right) \geq \delta, \quad \forall m \geq 1 .
$$

We recall that

$$
\mathscr{A}^{\alpha_{m}}=\bigcup_{g \in \mathscr{H}\left(g_{0}\right)} \mathscr{K}_{g}^{\alpha_{m}}(0) .
$$

Therefore, since $\psi_{m} \in \mathscr{A}^{\alpha_{m}}$, there exist $\sigma_{m} \in \mathscr{H}\left(g_{0}\right)$ and $v_{m} \in$ $\mathscr{K}_{\sigma_{m}}^{\alpha_{m}}$ such that $\psi_{m}=v_{m}(0)$.

Since $\left(t \mapsto v_{m}(t+h)\right) \in \mathscr{K}_{\sigma_{m}(\cdot+h)}^{\alpha_{m}} \forall h \in \mathbb{R}$, it follows that $v_{m}(t) \in \mathscr{A}^{\alpha_{m}} \subset B_{0} \forall t \in \mathbb{R}$. Since $B_{0}$ is an absorbing set for the process $\mathcal{U}_{\sigma_{m}}^{\alpha_{m}}(t, \tau)$ (see (51)), we have

$$
\left|v_{m}(t)\right|^{2} \leq 2 R_{0}^{2}
$$

where $R_{0}$ is independent of $m$ and $\alpha\left(\left\|\sigma_{m}\right\|_{L_{b}^{2}(\mathbb{R} ; H)}^{2} \leq\right.$ $\left.\left\|g_{0}\right\|_{L_{b}^{2}(\mathbb{R} ; H)}^{2}\right)$. Also, since $\mathscr{H}\left(g_{0}\right)$ is compact in $L_{\text {loc }}^{2}(\mathbb{R} ; H)$ and $\left\{\sigma_{m}\right\} \subset \mathscr{H}\left(g_{0}\right)$, there exists a subsequence of $v_{m}$ and $g \in$ $\mathscr{H}\left(g_{0}\right)$ such that

$$
\sigma_{m} \rightarrow g \text { in } \mathscr{H}\left(g_{0}\right) .
$$

Using the fact that each bounded sequence in a reflexive Banach space has a weakly convergent subsequence (see [20], Theorem 21.D, p. 255) and the boundedness (101), we deduce that

$$
v_{m}(t) \text { converges weakly in } H \text {. }
$$

Then, using the standard Cantor diagonal procedure as in [8, $15,16]$, we can deduce a function $\phi(s), s \in \mathbb{R}$, and a sequence $\left\{m_{j}\right\}$ such that

$$
v_{m_{j}}(t) \rightarrow \phi(t) \quad \text { weakly in } H \text { as } \quad j \longrightarrow \infty \text {. }
$$

From Proposition 11, we have that $\phi$ is a weak solution of the 2D Navier-Stokes equations. For $t=0$, we have

$$
\psi_{m_{j}} \rightarrow \phi(0) \text { in } H \text {. }
$$

Using the fact that $\mathscr{A}^{\alpha_{m}} \subset B_{1}$, where $B_{1}$ is given by (53) $\left(B_{1}\right.$ is uniformly absorbing set), we have

$$
\psi_{m_{j}} \longrightarrow \phi(0) \text { in } H,
$$

since $\psi_{m_{j}}$ is bounded in $V$. Also, since $\mathscr{A}_{0}=\bigcup_{g \in \mathscr{H}\left(g_{0}\right)} \mathscr{K}_{g}(0)$, we get $\phi(0) \in \mathscr{K}_{g}(0) \subset \mathscr{A}_{0}$. Passing to the limit in (99), we obtain $\delta=0$; and this contradicts the fact that $\delta>0$. This ends the proof of the theorem.

\section{Conflicts of Interest}

The author declares that there are no conflicts of interest regarding the publication of this paper.

\section{References}

[1] T. Tachim Medjo, "A non-autonomous two-phase flow model with oscillating external force and its global attractor," Nonlinear Analysis, vol. 75, no. 1, pp. 226-243, 2012.

[2] V. V. Chepyzhov, V. Pata, and M. I. Vishik, "Averaging of 2D Navier-Stokes equations with singularly oscillating forces," Nonlinearity, vol. 22, no. 2, pp. 351-370, 2009.

[3] V. V. Chepyzhov and M. I. Vishik, "Non-autonomous 2D Navier-Stokes system with singularly oscillating external force and its global attractor," Journal of Dynamics and Differential Equations, vol. 19, no. 3, pp. 655-684, 2007.

[4] S. Lu, "Attractors for nonautonomous 2D Navier-Stokes equations with less regular normal forces," Journal of Differential Equations, vol. 230, no. 1, pp. 196-212, 2006.

[5] S. Lu, H. Wu, and C. Zhong, "Attractors for nonautonomous 2D Navier-Stokes equations with normal external forces," Discrete and Continuous Dynamical Systems. Series A, vol. 13, no. 3, pp. 701-719, 2005.

[6] H. Song, S. Ma, and C. Zhong, "Attractors of non-autonomous reaction-diffusion equations," Nonlinearity, vol. 22, no. 3, pp. 667-681, 2009.

[7] P. E. Kloeden and B. Schmalfuss, "Non-autonomous systems, cocycle attractors and variable time-step discretization," Numerical Algorithms, vol. 14, no. 1-3, pp. 141-152, 1997.

[8] G. Yue and C. Zhong, "On the convergence of the uniform attractor of the 2D NS- $\alpha$ model to the uniform attractor of the 2D NS system," Journal of Computational and Applied Mathematics, vol. 233, no. 8, pp. 1879-1887, 2010.

[9] A. Haraux, "Systémes dynamiques dissipatifs et applications," in Recherches en Mathématiques Appliqueés, vol. 17, Mason, Paris, 1991.

[10] V. V. Chepyzhov and M. I. Vishik, in Attractors for equations of mathematical physics, vol. 49, American Mathematical Society Colloquium Publications, American Mathematical Society, Providence, RI, USA, 2002.

[11] R. Temam, Infinite Dynamical Dimensional Dynamical Systems in Mechanics and Physics, vol. 68, Springer-Verlag, New York, NY, USA, 2nd edition, 1988.

[12] A. V. Babin and M. I. Vishik, "Attractors of evolutions equations," in Studies in Mathematics and Its Applications, vol. 25, North-Holland, Publishing Co, Amsterdam, The Netherlands, 1992.

[13] E. Lunasin, S. Kurien, and E. S. Titi, "Spectral scaling of the Leray- $\alpha$ model for the two-dimensional turbulence," Journal of Physics. A. Mathematical and Theoretical, vol. 41, Article ID 344014, 2008.

[14] A. Cheskidov, D. D. Holm, E. Olson, and E. S. Titi, "On a Leray- $\alpha$ model of turbulence," Proceedings of The Royal Society of London. Series A. Mathematical, Physical and Engineering Sciences, vol. 461, pp. 629-649, 2005.

[15] V. V. Chepyzhov, E. S. Titi, and M. I. Vishik, "On the convergence of solutions of the Leray- $\alpha$ model to the trajectory attractor of the 3D Navier-stokes system," Discrete and Continuous Dynamical Systems, vol. 17, no. 3, pp. 481-500, 2007. 
[16] V. V. Chepyzhov, E. S. Titi, and M. I. Vishik, "On convergence of trajectory attractors of the 3D Navier-Stokes- $\alpha$ model as $\alpha$ approaches," Sb. Math, vol. 198, pp. 1703-1736, 2007.

[17] H. Bessaih and P. A. Razafimandimby, "On the rate of convergence of the $2 \mathrm{D}$ stochastic Leray- $\alpha$ model to the $2 \mathrm{D}$ stochastic Navier-Stokes equations with multiplicative noise," Applied Mathematics and Optimization, vol. 74, no. 1, pp. 1-25, 2016.

[18] Y. Cao and E. S. Titi, "On the rate of convergence of the two-dimensional $\alpha$-models of turbulence to the Navier-Stokes equations," Numerical Functional Analysis and Optimization, vol. 30, no. 11-12, pp. 1231-1271, 2009.

[19] A. A. Ilyin and E. S. Titi, "Attractors for the two-dimensional Navier-Stokes- $\alpha$ model: an $\alpha$-dependence study," Journal of Dynamics and Differential Equations, vol. 14, pp. 751-778, 2003.

[20] E. Zeidler, Nonlinear Functional Analysis and Its applications II/A: Linear Monotone Operators, Springer-Verlag, New York, NY, USA, 1990.

[21] J. L. Lions, Quelques Méthodes de Résolution des Problèmes aux Limites Non Linéaires, Dunod, Paris, Farnce, 1969. 


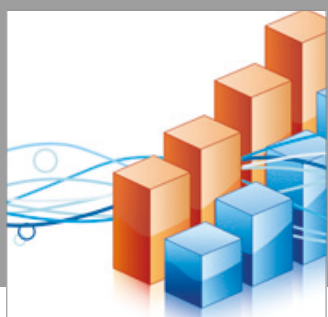

Advances in

Operations Research

vatersals

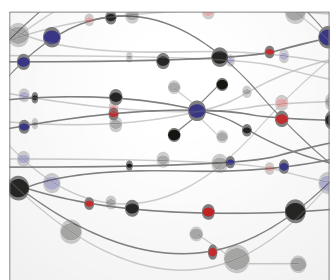

\section{The Scientific} World Journal
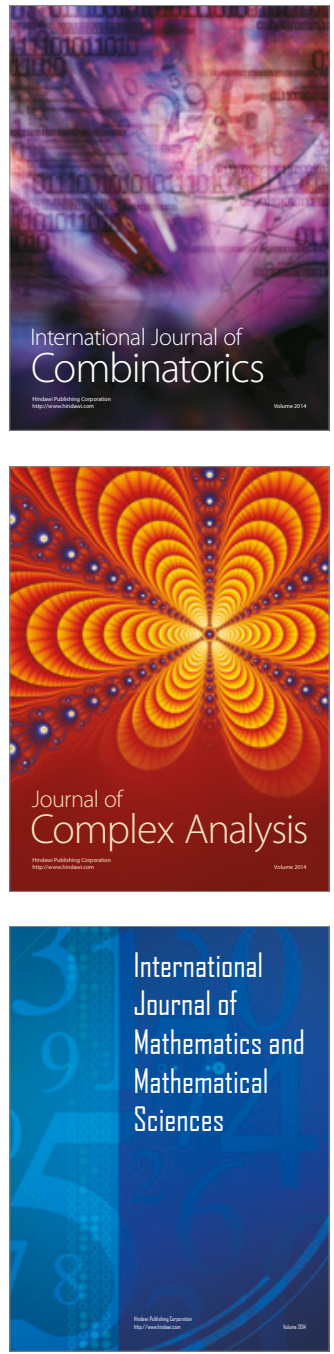
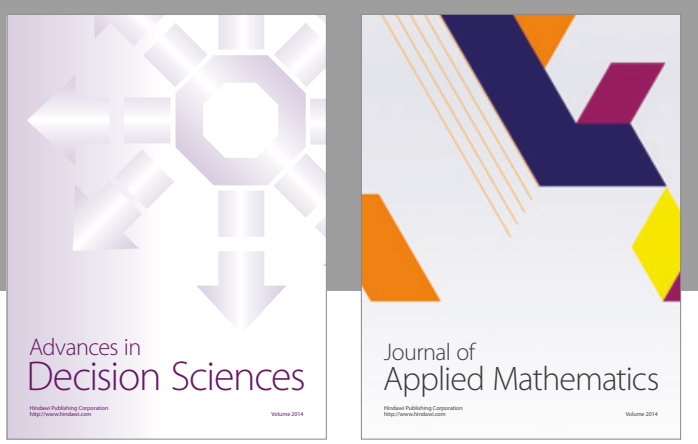

Algebra

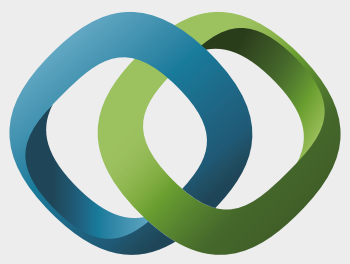

\section{Hindawi}

Submit your manuscripts at

https://www.hindawi.com
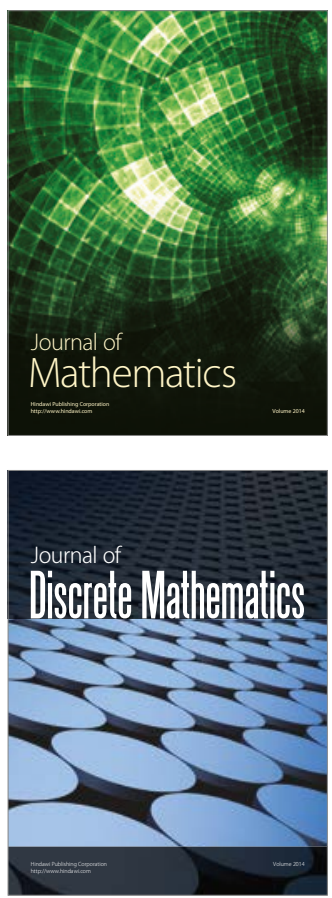

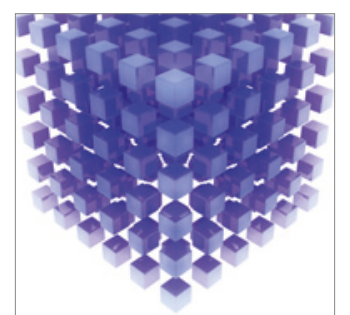

Mathematical Problems in Engineering
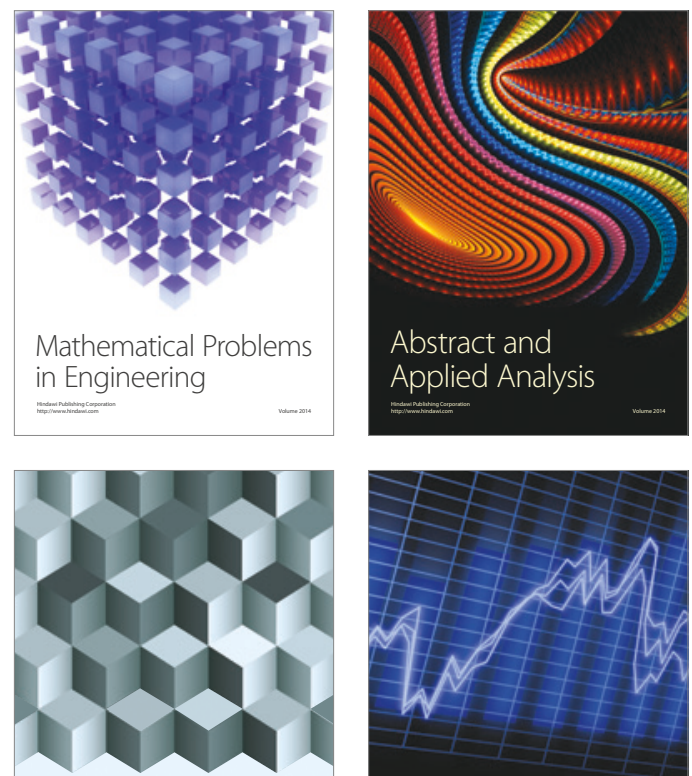

Journal of

Function Spaces

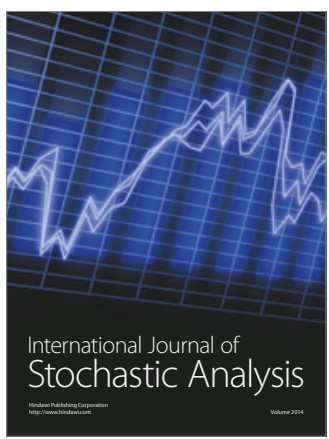

Probability and Statistics
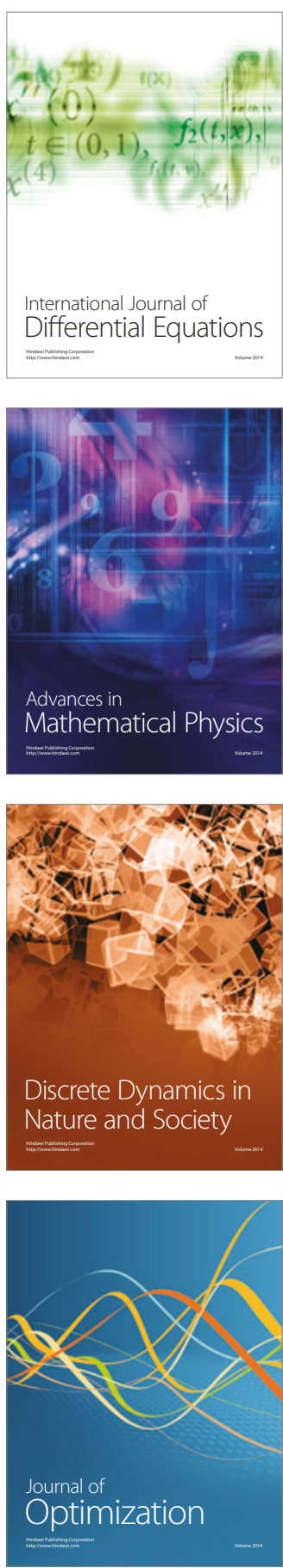